\title{
Indirect detection of a light Higgsino motivated by collider data
}

\author{
Katherine Freese* \\ Randall Physics Laboratory, University of Michigan, Ann Arbor, Michigan 48109-1120 \\ Marc Kamionkowski ${ }^{\dagger}$ \\ Department of Physics, Columbia University, 538 West 120th St., New York, New York 10027
}

(Received 16 September 1996)

\begin{abstract}
Kane and Wells recently argued that collider data point to a Higgsino-like lightest supersymmetric partner which would explain the dark matter in our Galactic halo. They discuss direct detection of such dark-matter particles in laboratory detectors. Here, we argue that such a particle, if it is indeed the dark matter, might alternatively be accessible in experiments which search for energetic neutrinos from dark-matter annihilation in the Sun. We provide accurate analytic estimates for the rates which take into account all relevant physical effects. Currently, the predicted signal falls roughly one to three orders of magnitude below experimental bounds, depending on the mass and coupling of the particle; however, detectors such as MACRO, superKamiokande, and AMANDA will continue to take data and should be able to rule out or confirm an interesting portion of the possible mass range for such a dark-matter particle within the next five years.

[S0556-2821(97)00304-4]
\end{abstract}

PACS number(s): 95.35.+d, 14.80.Ly, 95.55.Vj

\section{INTRODUCTION}

Recently, Kane and Wells (KW) proposed a Higgsino-like supersymmetric (SUSY) particle as a cold-dark-matter candidate [1]. This proposal was motivated [2] by a supersymmetric interpretation of the Collider Detector at Fermilab (CDF) Collaboration $e e \gamma \gamma+\mathbb{E}_{T}$ event [3] and/or the reported $Z \rightarrow b \bar{b}$ excess at the CERN $e^{+} e^{-}$collider LEP. In general, the lightest SUSY particle (LSP) is stable (if $R$ parity is conserved), and the other (heavier) SUSY particles eventually decay to the LSP. KW suggest the following explanation of the CDF event: $\widetilde{e}^{+}\left(\rightarrow e^{+} \chi_{2}\right) \widetilde{e}^{-}\left(\rightarrow e^{-} \chi_{2}\right)$, followed by the photinolike second-lightest neutralino $\chi_{2}$ decaying radiatively into the lightest neutralino (and LSP) $\chi$ and a photon. [Note that for this interpretation to work, the gaugino mass parameters must satisfy $M_{1} \sim M_{2}$ rather than the gauge unification condition $M_{1}=(5 / 3) \tan ^{2} \theta_{W} M_{2}$.] At present there has been only one such event, so that it is premature to claim discovery of supersymmetry. However, the evidence is certainly intriguing. It is therefore interesting to investigate the feasibility of discovering this particular dark-matter candidate with existing and forthcoming detectors. The hope is to either rule out this candidate or to detect it very soon. The payoff, namely, the discovery of supersymmetry and of the dark matter, would be enormous.

The proposed dark-matter candidate is Higgsino like:

$$
\chi \sim \sin \beta \widetilde{H}_{d}^{0}+\cos \beta \widetilde{H}_{u}^{0}+\delta \widetilde{Z},
$$

with $\delta<0.1$. In the models they consider, the LSP interactions with light fermions $f$ are due primarily to $Z^{0}$ exchange and are therefore approximated well by a $\chi \chi \bar{f} f$ low-energy effective Lagrangian with a coupling proportional to $\cos 2 \beta$,

\footnotetext{
*Electronic address: freese@mich.physics.lsa.umich.edu

†Electronic address: kamion@phys.columbia.edu
}

where $\tan \beta$ is the ratio of Higgs vacuum expectation values. The dark-matter phenomenology can therefore be parametrized simply by the LSP mass $m_{\chi}$ and $\cos 2 \beta$. In order for the SUSY explanation of the CDF $e$ e $\gamma \gamma+\boldsymbol{E}_{T}$ event to work, the LSP mass should be in the range $30 \mathrm{GeV} \leq m_{\chi}$ $\$ 55 \mathrm{GeV}$, and the radiative decay of the second-to-lightest neutralino requires $\tan \beta<2$ [2]. The model is consistent with constraints to the $Z^{0}$ invisible width [4] if $\tan \beta$ is further restricted to be even closer to unity or, equivalently, $\cos 2 \beta$ closer to zero. The cosmological abundance of the LSP is inversely proportional to its annihilation cross section. In this model, annihilation occurs predominantly through an $s$-channel $Z^{0}$ exchange, and so the annihilation cross section is proportional to $\cos ^{2} 2 \beta$ and depends also on the mass. In the regime of supersymmetric parameter space studied prior to the work of Kane and Wells, Higgsinos were not found to be viable dark-matter candidates because they annihilated too efficiently in the early Universe to retain a relic density of any significance. Here, on the other hand, the smaller values of $\tan \beta$ for this candidate lead to a smaller annihilation cross section and hence to a cosmologically interesting relic abundance (note that there is also no coannihilation with charginos in this case since the Higgsinos are much lighter than the charginos). The requirement that $\Omega_{\chi} h^{2} \lesssim 1$ (which derives from a conservative lower limit of $10 \mathrm{Gyr}$ to the age of the Universe) fixes $\cos ^{2} 2 \beta \geq 0.002$. Finally, although the $\mathrm{CDF}$ event does not require it, the $Z \rightarrow b \bar{b}$ anomaly favors a value of $m_{\chi} \lesssim 40 \mathrm{GeV}$. The range of models considered by KW which satisfy these constraints is shown in their Fig. 1. The parameter space is restricted primarily by their favored range for the relic abundance $0.1 \leqq \Omega_{\chi} h^{2} \leqq 0.5$ and by the $Z^{0}$ invisible width. This leaves an irregularly shaped region of favored parameter space which spans roughly the mass range $30 \mathrm{GeV} \lesssim m_{\chi} \lesssim 40 \mathrm{GeV}$ and $1.05 \leqq \tan \beta \leq 1.4$ or, equivalently, $0.002 \leq \cos ^{2} 2 \beta \leq 0.11$. 
KW studied the prospects for direct detection [5] of these particles in laboratory dark-matter detectors and found them promising for next-generation detectors [6]. In this paper, we point out that for the Higgsino-like particle they consider, searches for energetic neutrinos from LSP annihilation in the Sun [7] may be an equally or more promising avenue toward detection. The point is that the Higgsinos they consider have primarily axial-vector (rather than scalar) couplings to nuclei, and so they couple to the spin (rather than the mass) of a nucleus. The isotopic fraction of terrestrial nuclei with spin is generally small. On the other hand, about $75 \%$ of the mass of the Sun is composed of nuclei with spin (i.e., protons). Although detection of weakly interacting massive particles (WIMP's) with scalar interactions is probably more promising with laboratory detectors, detection of WIMP's with axial-vector interactions is generally more promising with astrophysical-neutrino detectors [8-10]. For example, Dirac neutrinos, which have scalarlike interactions, are currently ruled out as the Galactic dark matter over most of the plausible mass range by direct-detection experiments [11]. On the other hand, Majorana neutrinos, which have axial-vector interactions, are ruled out over a large mass range by null searches at Kamiokande [12], but are quite inaccessible to direct searches [10]. In fact, Rich and Tao found that the current bounds to the axial-vector WIMP-nucleon interaction strength from energetic-neutrino searches were roughly three orders of magnitude stronger than current limits from direct searches for WIMP's with masses near 30-40 GeV [10]. In this paper we consider both indirect and direct detection of the newly proposed [1] Higgsino dark-matter candidate.

In Sec. II, we calculate the energetic-neutrino rates and discuss the prospects for indirect detection. In Sec. III, we review the prospects for direct detection. In Sec. IV, we discuss the results and make some concluding remarks.

\section{ENERGETIC-NEUTRINO RATES}

If these particles are indeed present with a halo density $\rho_{\chi} \sim 0.3 \mathrm{GeV} \mathrm{cm}^{-3}$, then some passing through the Sun would lose enough energy to be captured. They then sink to the core of the Sun, and there build up enough density to start annihilating with each other. Among the annihilation products are ordinary neutrinos which would be observable in various existing detectors here on Earth. The energies of the neutrinos have a broad distribution centered roughly at a third of the LSP mass. The detectors with data already taken include Kamiokande [12], IMB [13], the Monopole, Astrophysics, and Cosmic Ray Observatory (MACRO) [14], Frejus [15], Baksan [16], and those being deployed now include the Antarctic Muon and Neutrino Detector Array (AMANDA) [17], NESTOR [18], and super-Kamiokande. The best technique for inferring the existence of these neutrinos is as follows: Muon neutrinos interact in the rock outside of the detector, and give rise to upward-going muons which can be registered in the detector. (Note that both muon neutrinos as well as muon antineutrinos are produced by the annihilation; these give rise to upward-going muons and antimuons. Both have been included in all our calculations and estimates, and we use the words "neutrinos" and "muons" to refer to the sum of particles and antiparticles.) The muonenergy thresholds for IMB and MACRO are roughly $2 \mathrm{GeV}$ and $1.7 \mathrm{GeV}$ for Kamiokande, and roughly $1 \mathrm{GeV}$ for Baksan. At present, IMB and Kamiokande constrain the flux of energetic neutrinos from the Sun with energies $\gtrsim 2 \mathrm{GeV}$ to be

$$
\Gamma_{\text {det }} \leqslant 2.1 \times 10^{-2} \mathrm{~m}^{-2} \mathrm{yr}^{-1} .
$$

In addition to the muon neutrinos, there is a comparable flux of electron neutrinos produced by the Higgsino annihilation. For the relatively low energies considered here, the efficiency for detection of electron neutrinos may be comparable to that described above for muon neutrinos. Thus the sensitivity to energetic neutrinos from Higgsino annihilation could be improved if one takes both channels into account. In this paper we focus on limits on the Higgsino particle that can be obtained from considering the production of muons only. Note that the Earth is composed primarily of spinless nuclei, and so axially coupled WIMP's will not be captured, and we expect no energetic-neutrino signal from the Earth for this dark-matter candidate.

Calculation of the predicted flux of neutrino-induced muons is straightforward but lengthy. It must take into account the complete capture-rate calculation, which includes the elastic-scattering cross section and the proper kinematic factors, and the time scale for equilibration between capture and annihilation. The neutrinos will be produced by decays of $b$ and $c$ quarks and $\tau$ leptons to which the LSP's annihilate. An accurate calculation of the neutrino spectrum must take into account the branching ratios for annihilation into various final states, hadronization and slowing of heavy hadrons, the three-body fermion-decay kinematics, and slowing and absorption of neutrinos in the Sun $[19,20]$. If the LSP has only an axial-vector coupling to nuclei, the result for the flux of neutrino-induced muons (for a local halo density of $0.3 \mathrm{GeV} \mathrm{cm}^{-3}$ and velocity dispersion of $270 \mathrm{~km} \mathrm{~s}^{-1}$ ) can be written ${ }^{1}$ [Eq. (9.55) in Ref. [9]]

$$
\begin{aligned}
\Gamma_{\mathrm{det}}= & \left(1.65 \times 10^{-4} \mathrm{~m}^{-2} \mathrm{yr}^{-1}\right) \sigma_{40} \tanh ^{2}\left(t_{\odot} / \tau_{\odot}\right) \\
& \times\left(m_{\chi} / \mathrm{GeV}\right) S\left(m_{\chi} / m_{p}\right) \xi\left(m_{\chi}\right),
\end{aligned}
$$

where $\sigma_{40}$ is the cross section for LSP-proton elastic scattering due to axial-vector interactions in units of $10^{-40} \mathrm{~cm}^{2}$, $S\left(m_{\chi} / m_{p}\right)$ (where $m_{p}$ is the proton mass) is a kinematic suppression factor, $\tau_{\odot}$ is the capture-annihilation equilibration time scale and $t_{\odot}$ is the age of the Sun, and $\xi\left(m_{\chi}\right)$ is a measure of the second moment of the neutrino energy distribution. We now discuss each of these factors.

In the models we are considering, the Higgsino-quark interaction is due primarily to $Z^{0}$ exchange. The cross section for scattering from a nucleus of mass $m_{N}$ with an unpaired proton is approximated by [1]

$\sigma_{N}=\frac{2 m_{\chi}^{2} m_{N}^{2}}{\pi\left(m_{\chi}+m_{N}\right)^{2}} G_{F}^{2} \cos ^{2} 2 \beta\left[\lambda^{2} J(J+1)\right](\Delta d+\Delta s-\Delta u)^{2}$

\footnotetext{
${ }^{1}$ Note that there is a factor of $\xi\left(m_{\chi}\right)$ missing and the $\tanh \left(t_{\odot} / \tau_{\odot}\right)$ should be $\tanh ^{2}\left(t_{\odot} / \tau_{\odot}\right)$ in Eq. (9.55) in Ref. [9].
} 
for unpaired-neutron nuclei, switch $\Delta d$ and $\Delta u$ (our $\Delta d$ and $\Delta u$ are switched relative to those in KW). For the LSPproton elastic scattering cross section, we take $m_{N}=m_{p}$ (proton mass) and the Lande factor $\lambda^{2} J(J+1)=3 / 4$ in Eq. (4). Here the $\Delta q$ 's are the fraction of spin in the proton carried by each quark. Using values from a recent compilation [21] which give $\Delta d+\Delta s-\Delta u=1.24$, the LSP-proton elastic scattering cross section evaluates to $\sigma_{40} \simeq 340 \cos ^{2} 2 \beta$. Using Eqs. (9.21)-(9.22) in Ref. [9], the kinematic factor $S\left(m_{\chi} / m_{p}\right)$ falls in the range $0.5-0.6$ for LSP masses between 30 and $40 \mathrm{GeV}$. Furthermore, the equilibration time scale is given by [Eq. (9.8) in Ref. [9]]

$$
\frac{t_{\odot}}{\tau_{\odot}}=330\left(\frac{C}{s^{-1}}\right)^{1 / 2}\left(\frac{\left\langle\sigma_{A} v\right\rangle}{\mathrm{cm}^{3} \mathrm{~s}^{-1}}\right)^{1 / 2}\left(\frac{m_{\chi}}{10 \mathrm{GeV}}\right)^{3 / 4},
$$

where the capture rate is [Eq. (9.19) in Ref. [9]],

$$
C=\left(1.3 \times 10^{25} \mathrm{~s}^{-1}\right) \sigma_{40} S\left(m_{\chi} / m_{p}\right)\left(m_{\chi} / \mathrm{GeV}\right)^{-1} .
$$

Here, $\left\langle\sigma_{A} v\right\rangle$ is the thermally averaged annihilation cross section times relative velocity in the limit $v \rightarrow 0$ (i.e., the $s$-wave contribution). If the neutralino-quark interaction occurs predominantly via $Z^{0}$ exchange, the $v \rightarrow 0$ annihilation cross section is

$$
\left\langle\sigma_{A} v\right\rangle=\frac{G_{F}^{2} \cos ^{2} 2 \beta}{8 \pi} \sum_{f} c_{f} m_{f}^{2},
$$

where the sum is over the $\tau$ lepton and $b$ and $c$ quarks to which the Higgsinos annihilate predominantly, $m_{f}$ is the fermion mass, and $c_{f}$ is a color factor ( 3 for quarks and 1 for leptons). Equation (7) evaluates to $\left\langle\sigma_{A} v\right\rangle \simeq 4.42 \times 10^{-27}$ $\mathrm{cm}^{3} \mathrm{~s}^{-1} \cos ^{2} 2 \beta$. Inserting our expressions for the annihilation cross section and capture rate into Eq. (5), we get $t_{\odot} / \tau_{\odot} \simeq 2600\left(m_{\chi} / 35 \mathrm{GeV}\right)^{1 / 4} \cos ^{2} 2 \beta$. For $\cos ^{2} 2 \beta \geq 0.002$ and the Higgsino masses of interest, $t_{\odot} / \tau_{\odot} \gtrsim 5$, and so we may safely set $\tanh ^{2}\left(t_{\odot} / \tau_{\odot}\right)$ equal to unity.

Finally, there is $\xi\left(m_{\chi}\right)$, a measure of the second moment of the neutrino energy distribution. This depends on the branching ratios for LSP-LSP annihilation into various annihilation channels. It is explicitly given by

$$
\xi\left(m_{\chi}\right)=\sum_{F} B_{F}\left[3.47\left\langle N z^{2}\right\rangle_{F, \nu}\left(m_{\chi}\right)+2.08\left\langle N z^{2}\right\rangle_{F, \bar{\nu}}\left(m_{\chi}\right)\right]
$$

where the sum is over all final states to which the LSP's can annihilate, and $B_{F}$ is the branching ratio for annihilation to each channel, which can be calculated with Eq. (7). The $\left\langle N z^{2}\right\rangle_{F, i}$ is the second moment of the energy distribution (scaled by $m_{\chi}^{2}$ ) of neutrino type $i$ from final state $F$. Analytic expressions for $\left\langle N z^{2}\right\rangle$, which take into account hadronization, stopping of heavy hadrons, the three-body decay kinematics, and slowing and absorption of neutrinos as they pass through the Sun, are given in Ref. [20], and these are accurate for the low-mass Higgsinos considered here [22]. As indicated in Eq. (8), $\xi\left(m_{\chi}\right)$ depends on the annihilation branching ratios, and the range of possible values is indicated in Fig. 33 in Ref. [9]. For LSP's with masses in the range $30-40 \mathrm{GeV}, \xi$ takes on its largest value $(\sim 0.11)$ for annihilation into $\tau$ leptons $\left(B_{\tau}=1\right)$ and its smallest value $(\sim 0.034)$ for annihilation into $b$ quarks $\left(B_{b}=1\right)$. If, as assumed by $\mathrm{KW}$, annihilation occurs via the $Z^{0}$ and $\tan \beta$ is near unity, then $B_{F} \propto c_{f} m_{f}^{2}$ [cf., Eq. (7)]. Therefore, Higgsinos should annihilate primarily to $b$ quarks, and so we will take $\xi\left(m_{\chi}\right)=0.034$. It should be kept in mind, however, that there will always be some nonzero annihilation branch into $\tau$ leptons, and in some models, if annihilation via $t$ - and $u$-channel exchange of a stau is larger, it may be significant. More generally, one can approximate $\xi$ for arbitrary annihilation branching ratios by noting that annihilation always occurs almost entirely to $\tau$ leptons and $b$ and $c$ quarks (since the other quarks are so much lighter). Furthermore, the value one would obtain for $\xi$ for the case of annihilation predominantly into $c$ quarks (i.e., if for some reason $\left.B_{c}=1\right)$ is quite close to the value one obtains for the most likely case of annihilation predominantly to $b$ quarks (i.e., when $\left.B_{b}=1\right)$, especially at low energies. Therefore, for LSP's with masses near $35 \mathrm{GeV}$, and for arbitrary branching ratios to $\tau$ leptons and $b$ and $c$ quarks, we can write $\xi \simeq 0.11 B_{\tau}+0.034\left(1-B_{\tau}\right)$. Therefore, by taking $B_{\tau}=0$, we are using a conservative lower limit for $\xi$, and it could conceivably be a factor of 3 larger.

Putting together all the factors, the detection rate is given by

$$
\begin{aligned}
\Gamma_{\mathrm{det}} \simeq & \left(2.7 \times 10^{-2} \mathrm{~m}^{-2} \mathrm{yr}^{-1}\right)\left(m_{\chi} / 35 \mathrm{GeV}\right) \\
& \times \cos ^{2} 2 \beta\left(\frac{\Delta d+\Delta s-\Delta u}{1.24}\right)^{2}\left[3.2 B_{\tau}+\left(1-B_{\tau}\right)\right] \\
& \times\left[\frac{S\left(m_{\chi} / m_{p}\right)}{0.55}\right] \tanh ^{2}\left[2600\left(m_{\chi} / 35 \mathrm{GeV}\right)^{1 / 4} \cos ^{2} 2 \beta\right],
\end{aligned}
$$

where we have included the dependence on the model parameters $m_{\chi}$ and $\cos ^{2} 2 \beta$, spin content of the proton, on the annihilation branch to $\tau$ leptons, the kinematic factor, and the equilibration time scale, although as indicated above the dependence on these last two factors will be very weak. Equation (9) is obtained assuming no muon energy thresholds (i.e., all muons can be detected); this assumption is a good approximation for detectors with thresholds near a few $\mathrm{GeV}$ (e.g., MACRO, Kamiokande, and Baksan), since these energies are negligible compared with the Higgsino mass. However, for detectors with higher thresholds (e.g., AMANDA and NESTOR), a good fraction of the signal may be below threshold.

The relic density is inversely proportional to $\cos ^{2} 2 \beta$. Since the count rate scales as $\cos ^{2} 2 \beta$, for a given mass the rate drops with increasing $\Omega_{\chi} h^{2}$. Furthermore, for a given relic abundance, the rate drops with increasing mass (see Fig. 1 in KW for the dependence of $\tan \beta$ on mass and abundance.) As an example, for $\Omega_{\chi} h^{2}=0.3$, the predicted count rates are $\Gamma_{\mathrm{det}} \sim 2.6 \times 10^{-3}$ for $m_{\chi}=30 \mathrm{GeV}$ and $\Gamma_{\mathrm{det}} \sim 1.1$ $\times 10^{-3}$ for $m_{\chi}=35 \mathrm{GeV}$.

In general, if, as is likely, annihilation occurs predominantly to $b$ quarks, then the predicted rates fall roughly one to three orders of magnitude below currently published limits for the $\cos ^{2} 2 \beta$ range of interest, $0.002 \leqq \cos ^{2} 2 \beta \leqq 0.11$. However, the accumulated exposure of Baksan is greater than that 
of Kamiokande, and so even better sensitivities (perhaps by a factor of 2) have probably already been achieved. Also, by performing an analysis of the data which takes into account the predicted angular and energy distribution of the neutrinoinduced muons, one should be able to improve the sensitivity with existing data [23]. More significantly, the MACRO Collaboration expects to reach a sensitivity a factor of 10 or so better than current bounds within the next five years as it continues to take data.

Future detectors such as super-Kamiokande, AMANDA, and NESTOR may have exposures orders of magnitude larger than current detectors. Whether they can improve on current sensitivities to light Higgsinos will depend on the thresholds of these detectors. These future detectors may have much larger thresholds (e.g., $10-30 \mathrm{GeV}$ ) so that the calculations described above would have to be redone. Although the neutrino energy distribution is centered roughly at a third the LSP mass, the probability of detecting a neutrino is proportional to the square of the neutrino energy. Therefore, the upward-muon signal is due to a large extent to the high-energy tail of the neutrino distribution. If so, there may still be a significant signal even for thresholds as high as $10-30 \mathrm{GeV}$, depending on the model parameters. In addition, for larger thresholds, the annihilation channel to $\tau$ particles via intermediate staus becomes important because the neutrinos produced via this channel are stiffer and hence capable of being above these larger thresholds.

\section{PROSPECTS FOR DIRECT DETECTION}

Let us now briefly review the prospects for direct detection in a laboratory detector, the ${ }^{73} \mathrm{Ge}$ detector, for example [1]. The rates are controlled by the cross section for Higgsino elastic scattering from a ${ }^{73} \mathrm{Ge}$ nucleus. (Note that the scattering rate from the naturally abundant isotope ${ }^{76} \mathrm{Ge}$ is very small since this isotope has no spin.) The cross section for scattering from a nucleus with an unpaired neutron, such as

${ }^{73} \mathrm{Ge}$, was given in Eq. (4) (with $\Delta d$ and $\Delta u$ reversed). In the single-particle shell model, the Lande factor for ${ }^{73} \mathrm{Ge}$ evaluates to $\lambda^{2} J(J+1) \simeq 0.3$. However, it should be kept in mind that the odd-group model predicts a number $80 \%$ smaller and more detailed calculations suggest it may be $2 \%$ [24] to $40 \%$ [25] smaller than in the single-particle shell model. Using the simplest (and most optimistic) value, Eq. (4) evaluates to $\sigma_{73}^{40} \simeq 6.5 \times 10^{4} \cos ^{2} 2 \beta\left[\lambda^{2} J(J+1) / 0.3\right]$, in units of $10^{-40} \mathrm{~cm}^{2}$, for Higgsinos with masses $30-40 \mathrm{GeV}$.

In order of magnitude, the rate for scattering from ${ }^{73} \mathrm{Ge}$ is $R \sim f_{73} \sigma_{73} \rho v /\left(m_{\chi} m_{N}\right)$ where $\rho$ is the local halo density, $v$ is the halo velocity dispersion, and $f_{73}$ is the isotopic fraction of ${ }^{73} \mathrm{Ge}$ in the sample. A careful calculation must include the velocity distribution (and its yearly modulation) of halo dark-matter particles incident on the detector and the proper form-factor suppression for spin-dependent scattering from ${ }^{73} \mathrm{Ge}[24,25]$. For a Higgsino of mass $40 \mathrm{GeV}$, the event rate (including all relevant physical effects) for scattering in ${ }^{73} \mathrm{Ge}$ may be obtained from the differential event rate for detection of an axially coupled WIMP plotted in Fig. 22 in Ref. [9]. The result (averaged over the yearly modulation) for scattering in natural germanium $\left(f_{73}=0.078\right)$ is

$$
\begin{aligned}
R & \simeq\left(1.2 \times 10^{-5} \mathrm{~kg}^{-1} \mathrm{day}^{-1}\right) f_{73} \sigma_{73}^{40} \\
& \simeq 0.0624 \cos ^{2} 2 \beta\left[\lambda^{2} J(J+1) / 0.3\right] .
\end{aligned}
$$

This result agrees (well within the nuclear-physics uncertainties) with the results shown in Fig. 2 in KW, although our numbers are slightly smaller. Equation (10) is obtained assuming no thresholds. However, finite energy thresholds in realistic experiments will cut out a significant fraction of events and lower the predicted detection rate accordingly.

Now consider, for illustration, the cryogenic dark matter search (CDMS) experiment [26], which will first run with $1 \mathrm{~kg}$ of natural germanium. After background rejection from demanding ionization-calorimetry coincidence, there will still be a background event rate of roughly $R_{b} \simeq 1 \mathrm{~kg}^{-1}$ day $^{-1}$. After a 1-yr exposure $(E=365 \mathrm{~kg}$ day $)$, the $3 \sigma$ sensitivity of the experiment will be roughly $S \simeq 3 \sqrt{R_{b} / E} \simeq 0.16 \mathrm{~kg}^{-1} \mathrm{day}^{-1}$. They also plan to run a similar experiment with roughly $0.5 \mathrm{~kg}$ of enriched ${ }^{73} \mathrm{Ge}$. Assuming the same background-event rate, this would improve the sensitivity to scattering from ${ }^{73} \mathrm{Ge}$ to 0.017 $\mathrm{kg}^{-1}$ day ${ }^{-1}$ when compared with the prediction above [cf. Eq. (10)] for scattering in natural germanium.

For $0.002 \leq \cos ^{2} 2 \beta \leq 0.11$, the predicted rate for Higgsino scattering in natural germanium is 0.0001-0.007 $\mathrm{kg}^{-1}$ day ${ }^{-1}$ (again, this is only slightly smaller than the results shown in Fig. 2 of $\mathrm{KW}$ ). Therefore, even the most optimistic models (with the most optimistic nuclear-physics and energy-threshold assumptions) seem to fall roughly a factor of 2 below this forecasted CDMS sensitivity.

\section{DISCUSSION}

The predicted rates for both direct [Eq. (10)] and indirect [Eq. (9)] detection of axially coupled WIMP's are proportional to the WIMP-nucleon coupling-in this case, $\cos ^{2} 2 \beta$. For a given WIMP mass, we can therefore compare the forecasted enriched- ${ }^{73} \mathrm{Ge}$ CDMS sensitivity with the current upward-muon limit. Doing so, we find that the enriched- ${ }^{73} \mathrm{Ge}$ sensitivity $\left(0.017 \mathrm{~kg}^{-1}\right.$ day $\left.{ }^{-1}\right)$ will improve on the current limit to the upward-muon flux $\left(2.1 \times 10^{-2}\right.$ $\mathrm{m}^{-2} \mathrm{yr}^{-1}$ ) roughly by a factor of 4 . When we compare this with the forecasted factor-of-10 improvement expected in MACRO, it appears that the sensitivity of indirect-detection experiments looks favorable. Before drawing any conclusions, however, it should be noted that the sensitivity in detectors with other nuclei with spin may be significantly better. We therefore conclude that the two schemes will be competitive for detection of axially coupled WIMP's. Realistically, we must also emphasize that the forecasted sensitivities of these current experiments will probe only the most optimistic region of KW's favored parameter space. It will require much larger low-background laboratory detectors or astrophysical-neutrino observatories to probe a good fraction of the interesting light-Higgsino models.

We should reemphasize that our estimates for both directand indirect-detection rates may actually be conservative. If our halo is flattened (as halos of many spirals seem to be), then the local halo density could be twice as large. If the LSP has a considerable annihilation branch to $\tau$ leptons, the neutrino rates could be up to a factor of 3 larger. It should also 
be kept in mind that-although KW argued that they should be small-the LSP-quark interaction will have some squarkexchange contributions in addition to the $Z^{0}$ contributions. This will probably expand the viable parameter space. It also implies that there may realistically be additional contributions to the axial-vector coupling to nuclei, and there may be some scalar interaction. This could enhance rates for both direct and indirect detection, although much more dramatically for direct detection. It would also result in a neutrino signal from the center of the Earth.

There are also uncertainties which might reduce the rates. Variations within current experimental constraints in the spin content of the nucleon could either increase or decrease both direct- and indirect-detection rates, perhaps dramatically. The Lande factor is likely to be smaller than the value from the single-particle shell model which we used, and if so, the direct-detection rates will be lowered accordingly. There may be sizable errors in the form factor for spin-independent scattering which would affect the direct rates. Finally, we have assumed $100 \%$ direct-detection efficiency. However, for the Higgsino mass range of interest, a significant fraction of the recoils could be below the detection threshold, and the sensitivity to an LSP passing through the detector would be degraded accordingly.

Within the next five years, detectors at LEP and Fermilab will be able to confirm or rule out the $e e \gamma \gamma+\boldsymbol{E}_{T}$ event that motivated this Higgsino dark-matter candidate. Much of the available parameter space for the SUSY interpretation will in fact be tested within one year. If the SUSY interpretation of this event is indeed correct, then this Higgsino particle exists, but one still does not know how long it lives; the fact that it escapes the detector only proves that its lifetime is longer than $\sim 10^{-8} \mathrm{~s}$. Proving that this particle is in fact the dark matter in the halo of our Galaxy would require that it be detected either directly or indirectly due to its annihilation in the Sun. Of course these techniques may be used to rule out the existence of the particle instead. In this paper, we have suggested that the time scale for indirect detection may be comparable to that for experimental verification or disproof of the motivating event.

To conclude, we have provided estimates of the rates for indirect detection of Higgsino dark-matter candidates motivated by collider data. Our calculations take into account all relevant physical effects; the accuracy of our estimates should be well within the irreducible astrophysics and particle-physics uncertainties inherent in any such calculation. Our results-obtained assuming only a $Z^{0}$-exchange contribution to the LSP-quark interaction-suggest that indirect detection may provide a realistic alternative avenue toward verification or falsification of this Higgsino dark-matter candidate.

\section{ACKNOWLEDGMENTS}

We would like to thank D. Akerib, E. Diehl, R. Gaitskell, G. Kane, C. Kolda, and G. Tarle for helpful discussions. M.K. thanks the Theory Division at CERN for hospitality, and M.K. and K.F. thank the Fermilab Theoretical Astrophysics Center (where part of this work was completed) for hospitality. This work was supported at Columbia by the U.S. DOE under Contract No. DEFG02-92-ER 40699, NASA under Grant No. NAG5-3091, the Alfred P. Sloan Foundation, and at Michigan by Grant No. NSFPHY9407194. Portions of this work (K.F.) were completed at the Aspen Center for Physics.
[1] G. L. Kane and J. D. Wells, Phys. Rev. Lett. 76, 4458 (1996).

[2] S. Ambrosanio et al., Phys. Rev. Lett. 76, 3498 (1996).

[3] S. Park, in 10th Topical Workshop on Proton-Antiproton Collider Physics, edited by R. Raja and J. Yoh, AIP Conf. Proc. No. 357 (AIP, New York, 1996).

[4] LEP Collaborations, Report No. CERN-PPE/95-172 (unpublished).

[5] M. W. Goodman and E. Witten, Phys. Rev. D 31, 3059 (1985); I. Wasserman, ibid. 33, 2071 (1986); K. Freese, J. Frieman, and A. Gould, ibid. 37, 3388 (1988); K. Griest, ibid. 38, 2357 (1988); Report No. FERMILAB-Pub-89/139-A(E) (unpublished); A. Drukier, K. Freese, and D. Spergel, Phys. Rev. D 33, 3495 (1986).

[6] See, e.g., Proceedings of the Fifth International Workshop on Low Temperature Detectors, Berkeley, California, 1993 [J. Low Temp. Phys. 93, Nos. 3/4 (1993)].

[7] J. Silk, K. A. Olive, and M. Srednicki, Phys. Rev. Lett. 55, 257 (1985); K. Freese, Phys. Lett. 167B, 295 (1986); L. M. Krauss, K. Freese, D. N. Spergel, and W. H. Press, Astrophys. J. 299, 1001 (1985); L. M. Krauss, M. Srednicki, and F. Wilczek, Phys. Rev. D 33, 2079 (1986); T. Gaisser, G. Steigman, and S. Tilav, ibid. 34, 2206 (1986); K.-W. Ng, K. A. Olive, and M. Srednicki, Phys. Lett. B 188, 138 (1987); M. Kamionkowski, Phys. Rev. D 44, 3021 (1991).
[8] M. Kamionkowski, K. Griest, G. Jungman, and B. Sadoulet, Phys. Rev. Lett. 74, 5174 (1995).

[9] G. Jungman, M. Kamionkowski, and K. Griest, Phys. Rep. 267, 195 (1996).

[10] J. Rich and C. Tao, Report No. DAPNIA/SPP 95-01 (unpublished).

[11] M. Beck, in TAUP 93, Proceedings of the Third International Workshop on Theoretical and Phenomenological Aspects of Underground Physics, Assergi, Italy, edited by C. Arpesella et al. [Nucl. Phys. B, Proc. Suppl. 35, 150 (1994)]; M. Beck et al., Phys. Lett. B 336, 141 (1994); S. P. Ahlen et al., ibid. 195, 603 (1987); D. O. Caldwell et al., Phys. Rev. Lett. 61, 510 (1988).

[12] Kamiokande Collaboration, M. Mori et al., Phys. Lett. B 289, 463 (1992); Kamiokande Collaboration, M. Mori et al., Phys. Rev. D 48, 5505 (1993).

[13] IMB Collaboration, J. M. LoSecco et al., Phys. Lett. B 188, 388 (1987).

[14] E. Diehl, Ph.D. thesis, University of Michigan, 1994; E. Diehl, G. Kane, C. Kolda, and J. Wells, Phys. Rev. D 50, 4223 (1994).

[15] Frejus Collaboration, presented by H. J. Daum, Topical Seminar on Astrophysics and Particle Physics, San Miniato, Italy, 1989.

[16] M. M. Boliev et al., Izv. Akad. Nauk SSSR 55, 748 (1991) 
[Bull. Acad. Sci. USSR, Phys. Ser. 55, 126 (1991)].

[17] D. M. Lowder et al., Nature (London) 353, 331 (1991).

[18] L. Resvanis, Europhys. News 23, 172 (1992).

[19] S. Ritz and D. Seckel, Nucl. Phys. B304, 877 (1988).

[20] G. Jungman and M. Kamionkowski, Phys. Rev. D 51, 328 (1995).

[21] The Spin Muon Collaboration, D. Adams et al., Phys. Lett. B 329, 399 (1994).
[22] J. Edsjö (private communication).

[23] L. Bergstrom, J. Edsjö, and M. Kamionkowski (in preparation).

[24] M. T. Ressell et al., Phys. Rev. D 48, 5519 (1993).

[25] V. Dimitrov, J. Engel, and S. Pittel, Phys. Rev. D 51, 291 (1995).

[26] P. D. Barnes et al., J. Low Temp. Phys. 93, 79 (1993); T. Shutt et al., Phys. Rev. Lett. 69, 3425 (1992); 69, 3531 (1992). 